JiANHUI HUANG (Edmonton)

NA Li (Hong Kong)

\title{
VALUATION AND OPTIMAL DESIGN TO DEFAULTABLE SECURITY
}

Abstract. Herein, we develop a backward stochastic differential equation (BSDE) valuation of securities with default risk. Consequently, the optimal recovery problem with quasi-linear utility functions is discussed with the help of the stochastic maximum principle. Finally, two important examples: the exponential and power utility cases are studied and their business implications are considered.

1. Introduction. Credit risk (or default risk) has become the central interest of the financial community, with the development of new financial instruments exposed to default risk in financial engineering. Most of the extant credit risk literature concerns the determination of credit spread dynamics whereas the optimal design of defaultable securities has been traditionally neglected although it should deserve more attention. Our paper aims to fill this gap. We discuss the optimal design of corporate bond, precisely speaking, the optimal recovery problem which consists of seeking and characterizing the optimal recovery policy in bond issuance under the recovery of market value (RMV) scheme of Duffie and Singleton [1999].

One contribution of our work is in the category of default risk valuation which acts as the preliminary part to the credit risk analysis. Our modeling setup belongs to the reduced form framework which goes back to Artzner and Delbaen [1995], Jarrow and Turnbull [1995] and Duffie and Singleton [1999]. Under the RMV scheme, we develop a valuation representation of defaultable securities in terms of backward stochastic differen-

2000 Mathematics Subject Classification: 49K45, 60G35, 60H10.

Key words and phrases: default risk, reduced-form model, RMV, BSDE, stochastic maximum principle, quasi-linear utility.

We are grateful to Prof. Shige Peng for his valuable and insightful comments. We are also grateful for the useful comments of the anonymous referee. 
tial equations (BSDE) and our result can be extended to the more general forward-backward stochastic differential equation (FBSDE) case.

Another contribution of our work lies in the optimal security design, an indispensable building block to the overall credit risk management. Williams [2003] investigates the principle-agent problems in continuous time with stochastic control techniques and gives some sufficient condition for the optimal contract design. But to the best of our knowledge, this paper is the first research in optimization of debt financing within the dynamic recovery environment. We focus on a risk-neutral enterprise which aims to maximize its objective functional based on some time additive utilities. We transform this optimization problem into the framework of stochastic control by viewing the recovery rate in RMV as a controlled variable. Afterwards, we apply the stochastic maximum principle to derive the optimal control.

The rest of this paper is organized as follows. Section 2 introduces the basic economy setting in which the valuation and optimal design are discussed. Section 3 is devoted to the BSDE valuation of defaultable securities where the recovery rate is given. The optimal recovery problem as well as its optimal solution are spelled out in Section 4. Some further implications to negative exponential and power utilities are presented in Section 5. Section 6 concludes with some possible extensions.

2. Reduced form model. We consider a continuous time financial model defined on some filtered probability space $\left(\Omega, \mathcal{F}, \mathcal{F}_{t}, P\right)$ which satisfies the "usual conditions" (see Protter [1990] for technical details). The filtration $\mathcal{F}_{t}$ represents all the information that arrived to the market up to time $t$. A short-rate process $r_{t}$ is also fixed and assumed to be bounded and progressively measurable. The probability measure $P$ is assumed to be the equivalent martingale measure introduced by Harrison and Kreps [1979] and its existence is essentially equivalent to the arbitrage-free assumption on the market. Any defaultable security in this market, with maturity $T$, can be represented by a triple $(\xi, \Psi, \tau)$ where:

- $\xi$ is an $\mathcal{F}_{T}$-measurable random variable satisfying $E \xi^{2}<\infty$ and it represents the payoff received by the security holder at $T$ provided no default has occurred before. The default time $\tau$ is assumed to be an $\mathcal{F}_{t}$-stopping time.

- The process $\Psi_{t}$ stands for the amount of money received by the security holder if default occurs at some time $t<T$. Following Duffie and Singleton [1999], throughout this paper, we will assume the RMV scheme for $\Psi$, that is $\left(^{1}\right), \Psi_{t}=\left(1-L_{t}\right) Y_{t}$.

$\left({ }^{1}\right)$ Similar to Duffie and Singleton [1999], here we assume the price process admits no jumps, that is, $\Delta Y_{t}=0$. 
- The fraction loss rate $L_{t}$ is a predictable $\left({ }^{2}\right)$ process and because in no situation, can the security holder lose more than the security's total market value $Y_{t}$, we have $L_{t} \leq 1$. However, $L_{t}$ can take negative values as explained later.

REMARK 2.1. In principle, we can consider the "general recovery rule" suggested by Bielecki and Rutkowski [2002], where the payoff process $\Psi$ satisfies

$$
\Psi_{t}=p\left(t, Y_{t}\right)
$$

Here, $p(t, x)$ is Lipschitz continuous with respect to $t$ and $p(t, 0)=0$.

Given a defaultable security $(\xi, \Psi, \tau)$, its default process is defined as $N_{t}=1_{\{\tau \leq t\}}$, which is a stochastic process taking value 1 if default has occurred and 0 otherwise. Unlike the traditional structural models stemming from the pioneering work of Black and Scholes [1973] and Merton [1974], the reduced-form approach models the default process with a presumed default intensity process given below. As a standard result, the Doob-Meyer decomposition theorem enables us to decompose $N_{t}$ as

$$
N_{t}=A_{t}+M_{t}
$$

where the compensator $A_{t}$ should be some predictable increasing process and $M_{t}$ a martingale. Furthermore, suppose there exists some progressively measurable and nonnegative process $\lambda_{t}$ such that

$$
A_{t}=\int_{0}^{t \wedge \tau} \lambda_{u} d u=\int_{0}^{t} 1_{\{t \leq \tau\}} \lambda_{t} d u
$$

Then $\lambda_{t}$ is called the default intensity process (or hazard rate process) as it describes the conditional default rate, or intensity in the sense that conditional on no default prior to $t$, the likelihood of default during the time interval $(t, t+d t)$ is just $\lambda_{t} d t$. As the key feature distinguishing them from the structure model, in the reduced-form models, the default intensity $\lambda_{t}$ is assumed to be exogenous, in other words, the default is an unpredictable event depending on the whole economy situation instead of the issuer's market value itself.

3. BSDE valuation with given recovery rate. The main result in this section is that, under some mild conditions, the price process of $(\xi, \Psi, \tau)$ can be represented as the state solution of some BSDE provided the recovery rate is given. The BSDE, first introduced by Bismut [1973] for the linear

$\left({ }^{2}\right)$ See Protter [1990] for the definition. 
case and Pardoux and Peng [1990] for the general case, is

$$
\left\{\begin{array}{l}
-d Y_{t}=f\left(t, Y_{t}, Z_{t}\right) d t-Z_{t} d W_{t}, \\
Y_{T}=\xi
\end{array}\right.
$$

where $W$ is the standard Brownian motion with respect to $\mathcal{F}_{t}$ and the functional $f$ is always called the driver of the BSDE. Unlike its counterpart in the "forward" stochastic differential equation (SDE), the solution to the BSDE has two components: the state solution $Y$ and volatility solution $Z$, where the additional component $Z$ is just the risk adjustment factor in hedging theory.

THeOREM 3.1. Under the general recovery rule, suppose $p(t, x)$ is Lipschitz continuous with respect to $t$, and $r_{t}, \lambda_{t}$ are bounded and progressively measurable processes. Then the price process $Y_{t}$ of $(\xi, \Psi, \tau)$ satisfies the following BSDE:

$$
\left\{\begin{array}{l}
d Y_{t}=\left[\left(r_{t}+\lambda_{t}\right) Y_{t}-p\left(t, Y_{t}\right) \lambda_{t}\right] d t+Z_{t} d W_{t}, \\
Y_{T}=\xi
\end{array}\right.
$$

Proof. The defaultable security $(\xi, \Psi, \tau)$ can be written uniformly as $D_{t}$ where

$$
D_{t}= \begin{cases}\Psi_{\tau} 1_{\{\tau \leq t\}}, & t<T, \\ \Psi_{\tau} 1_{\{\tau \leq T\}}+\xi 1_{\tau>T}, & t \geq T .\end{cases}
$$

Then recall the decomposition of the default process $N_{t}$ :

$$
N_{t}=1_{\{\tau \leq t\}}=A_{t}+M_{t}=\int_{0}^{t \wedge \tau} \lambda_{u} d u+M_{t}=\int_{0}^{t} 1_{\{u \leq \tau\}} \lambda_{u} d u+M_{t} .
$$

For $t>0$, we have

$$
D_{t}=\int_{0}^{t \wedge T} \Psi_{s} 1_{\{s \leq \tau\}} \lambda_{s} d s+\int_{0}^{t \wedge T} \Psi_{s} d M_{s}+\xi 1_{\{\tau>T, t \geq T\}} .
$$

From Harrison and Kreps [1979], the price process $Y_{t}$ of $(\xi, \Psi, \tau)$ should be

$$
Y_{t}=E\left[\int_{t}^{T} \exp \left(-\int_{t}^{u} r_{s} d s\right) \Psi_{u} 1_{\{u<\tau\}} \lambda_{u} d u+\exp \left(-\int_{t}^{T} r_{s} d s\right) \xi 1_{\{\tau>T\}} \mid \mathcal{F}_{t}\right] .
$$

Because $\Psi_{t}=p\left(t, Y_{t}\right)$, we have

$$
Y_{t}=E\left[\int_{t}^{T} \exp \left(-\int_{t}^{u} r_{s} d s\right) p\left(u, Y_{u}\right) 1_{\{u \leq \tau\}} \lambda_{u} d u+\exp \left(-\int_{t}^{T} r_{s} d s\right) \xi 1_{\{\tau>T\}} \mid \mathcal{F}_{t}\right] .
$$

From the nonlinear Feynman-Kac formula,

$$
Y_{t}=E\left[\int_{t}^{T}\left(p\left(u, Y_{u}\right) \lambda_{u}-r_{u} Y_{u}\right) d u+\xi 1_{\{\tau>T\}} \mid \mathcal{F}_{t}\right] .
$$


As discussed in Duffie, Schroder and Skiadas [1996], we can eliminate the indicator function $1_{\{\tau>T\}}$ and get the following recursive valuation formula:

$$
Y_{t}=E\left[\int_{t}^{T}\left[\left(p\left(u, Y_{u}\right)-Y_{u}\right) \lambda_{u}-r_{u} Y_{u}\right] d u+\xi \mid \mathcal{F}_{t}\right] .
$$

Now consider the following BSDE:

$$
\left\{\begin{array}{l}
d Y_{t}=\left[\left(r_{t}+\lambda_{t}\right) Y_{t}-p\left(t, Y_{t}\right) \lambda_{t}\right] d t+Z_{t} d W_{t}, \\
Y_{T}=\xi .
\end{array}\right.
$$

As we assumed $p(t, x)$ is Lipschitz continuous and $r_{t}, \lambda_{t}$ are both bounded and predictable, from Pardoux and Peng [1990], we know the above BSDE has a unique adapted solution $(Y, Z)$. If we write the above BSDE in integral form, we have

$$
Y_{t}=\xi-\int_{t}^{T}\left[\left(r_{t}+\lambda_{t}\right) Y_{t}-p\left(t, Y_{t}\right) \lambda_{t}\right] d t-\int_{t}^{T} Z_{t} d W_{t}
$$

After taking the conditional expectation, the last term vanishes due to the martingale property of Itô integration,

$$
Y_{t}=E\left[\int_{t}^{T}\left[\left(p\left(u, Y_{u}\right)-Y_{u}\right) \lambda_{u}-r_{u} Y_{u}\right] d u+\xi \mid \mathcal{F}_{t}\right] .
$$

This completes the proof.

As in the special case when RMV is assumed, $\Psi_{t}=p\left(t, Y_{t}\right)=\left(1-L_{t}\right) Y_{t}$, we have the more explicit result than that obtained in Duffie and Singleton [1999].

TheOREM 3.2. Under the RMV scheme, the price $Y_{t}$ of a defaultable claim $(\xi, \Psi, \tau)$ satisfies the following BSDE:

$$
\left\{\begin{array}{l}
d Y_{t}=\left(r_{t}+\lambda_{t} L_{t}\right) Y_{t} d t+Z_{t} d W_{t}, \\
Y_{T}=\xi .
\end{array}\right.
$$

The advantage of our BSDE valuation is that it gives a dynamical rather than static representation of the price behavior; and based on this, we can use the stochastic control techniques to solve the optimization problem recursively.

\section{Optimal security design}

4.1. Optimal recovery policy. Assume that a firm with a project prefers to choose debt as its financing strategy. Suppose it plans to issue a zerocoupon bond and commits to the RMV scheme where it can choose its recovery policy dynamically. To simplify the analysis, here we first provide a heuristic introduction of the objective functional in a discrete-time setting 
and then reformulate it in a continuous-time setting. Consider a discretetime market with $N$ time instants on which the firm's bond is introduced with payment $\xi$ at the expiry time $N$. Moreover, for $n=1, \ldots, N$ :

- If no default occurs before time $n$, then let $\lambda_{n}$ be the conditional probability of default between times $n$ and $n+1$.

- $\beta_{n}$ and $Y_{n}$ are respectively the discount-free short rate and the bond's market value at time $n$.

- $L_{n}$ is the recovery rate in case the default occurs at time $n$.

According to RMV, if the default really occurs at time $n$, the firm needs only pay $\left(1-L_{n}\right) Y_{n}$ to its bond holder. Because the bond's market value at time $n$ is $Y_{n}$, this recovery policy helps the firm save as much as $Y_{n}-\left(1-L_{n}\right) Y_{n}=$ $L_{n} Y_{n}$ compared to its default-free situation. Therefore, $L_{n} Y_{n}$ is the benefit to the firm due to the recovery policy $\left\{L_{n}\right\}_{n=1}^{N}$ and we call it the intertemporal gain process. Following Hojgaard and Taksar [2001], we can treat it as a controlled variable and consider the following functional in discrete time:

$$
J^{N}(L)=E\left[\sum_{n=1}^{N} e^{-\sum_{j=1}^{n} \beta_{j}} U_{1}\left(L_{n} Y_{n}\right) \lambda_{n}+U_{2}\left(Y_{0}\right)\right],
$$

where $U_{1}, U_{2}$ are two utility functions. Now we discuss its business implications:

- The terms $U_{1}\left(L_{n} Y_{n}\right), n=1, \ldots, N$, denote the firm's preference to instantaneous gain at time $n$. Although the default may occur at any time from 1 to $N$, it cannot occur at two different times simultaneously, as for a given bond, there is at most one default. Therefore, we should take account of these terms in a somewhat "average" sense by alternatively considering $U_{1}\left(L_{n} Y_{n}\right) \cdot \lambda_{n}$ for each $n=1, \ldots, N$, where the "weight" $\lambda_{n}$ is included here to represent their respective likelihoods. Two of the most typical examples will be respectively: (1) $\lambda_{n} \equiv \lambda$ where $\lambda$ is some constant; (2) $\lambda_{n}$ is some deterministic function on $n$. In general, our default process is "double stochastic", that is, $\lambda_{n}$ and $L_{n} Y_{n}$ are both stochastic processes (see Cox [1955] for the details). Thus, our objective functional still needs to involve the expectation under $P$ to capture such "double stochastic" feature. Therefore, on the whole, the first term in $J^{N}(L)$ measures the "cumulative" satisfaction to the instantaneous gain process $L_{n} Y_{n}$.

- $U_{2}\left(Y_{0}\right)$ denotes the firm's preference to the initial bond price $Y_{0}$ which is exactly the amount of money initially raised per share and it changes conversely to the bond's yield to maturity rate (YTM).

In the continuous-time case, given the recovery policy $1-L_{t}$ and utility functions $U_{1}, U_{2}, \lambda_{t} d t$ is just the likelihood of default during the infinitesimal 
time interval $(t, t+d t)$ if no default occurs prior to $t$. Therefore, similarly to the reasoning in the discrete-time case, we can define the objective functional

$$
J(L)=E\left[\int_{0}^{T} e^{-\int_{0}^{t} \beta_{s} d s} U_{1}\left(L_{t} Y_{t}\right) \lambda_{t} d t+U_{2}\left(Y_{0}\right)\right],
$$

which represents the firm's expected utility over its intertemporal gain process and the initial financing amount $Y_{0}$. Here, $\beta$ is the discount rate over time. The firm aims to maximize this time-additive objective functional $J(L)$ over the admissible recovery policy set $\mathcal{U}$ which we will specify soon.

For the sake of implementation and tractability, here we consider the objective functional defined on the claim's whole life horizon $[0, T]$. A more realistic objective functional, defined on a random time interval, is briefly discussed at the end of this subsection.

Intuitively, the lower $L_{t}$, the higher $Y_{0}$ (equivalently the lower YTM the investor required), hence the heavier the recovery obligation $\left(1-L_{t}\right) Y_{t}$ to the firm in case of default, and this will offset the benefits from the lower YTM somewhat. Conversely, the higher $L_{t}$, the lower $Y_{0}$, but on the other hand, the higher $L_{t}$ will release the firm's recovery burden on default. Therefore, on the whole, $J(L)$ trades off the firm's gratification between the instantaneous gain process and the initially raised capital, and the firm's objective can be formulated as the following optimal recovery problem in the setting of stochastic control. In the following, we will replace $L$ by $u$ to be consistent with the conventions of control theory.

Definition 4.1. The optimal recovery problem is to find

$$
\sup _{u \in \mathcal{U}} J(u)=\sup _{u \in \mathcal{U}} E\left[\int_{0}^{T} e^{-\int_{0}^{t} \beta_{s} d s} U_{1}\left(u_{t} Y_{t}\right) \lambda_{t} d t+U_{2}\left(Y_{0}\right)\right],
$$

equivalently $\left({ }^{3}\right)$

$$
\inf _{u \in \mathcal{U}}-J(u)=\inf _{u \in \mathcal{U}} E\left[\int_{0}^{T}-e^{-\int_{0}^{t} \beta_{s} d s} U_{1}\left(u_{t} Y_{t}\right) \lambda_{t} d t-U_{2}\left(Y_{0}\right)\right],
$$

where

$$
\left\{\begin{array}{l}
d Y_{t}=\left(r_{t}+\lambda_{t} u_{t}\right) Y_{t} d t+Z_{t} d W_{t}, \\
Y_{T}=\xi
\end{array}\right.
$$

and $\lambda_{t}>0, r_{t}$ are bounded and progressively measurable processes. The

$\left({ }^{3}\right)$ Note that $\sup _{u \in \mathcal{U}} J(u)=-\inf _{u \in \mathcal{U}}[-J(u)]$; we state the optimal recovery problem in this form to relate it directly to control theory which typically considers the minimization of the objective functional. 
admissible control set $\mathcal{U}$ is

$$
\mathcal{U}=\left\{u: E \int_{0}^{T} u^{2}(s) d s<\infty, u(\cdot) \in \mathcal{C}=(-\infty, 1) \text { a.e. }\right\} .
$$

Remark 4.2. We set the admissible control domain $\mathcal{C}$ to be $(-\infty, 1)$, so the loss rate $u$ can take a negative value. This is reasonable as, in some cases, the firm itself would like to compensate more than the bond market value to its bond holder to keep its business reputation.

The optimal recovery policy is the admissible control $u^{*} \in \mathcal{U}$ which maximizes the objective functional. If $Y^{*}$ is the price process corresponding to $u^{*}$, then $\left(u^{*}, Y^{*}\right)$ will be called the optimal control pair, a terminology borrowed from control theory.

In this paper, we focus on the situations where the utility functions $U_{1}, U_{2}$ are of the quasi-linear form of Hansen [1985] and Rogerson [1988]. The quasi-linear utility is one of the benchmark macroeconomic models and it is used to describe the preference to two different incomes $x, y$ through

$$
U(x, y)=U_{1}(x)+K y, \quad K>0 .
$$

That is, $U$ is additive in $x$ and $y$, and linear in $y$, where $U_{1}$ satisfies the Inada condition. In the optimal recovery problem, we assume the firm's preference to the initial price $Y_{0}$ is linear as in the quasi-linear case $\left(^{4}\right)$

$$
U_{2}\left(Y_{0}\right)=K Y_{0}, \quad K>0 .
$$

A nontrivial example is when $U_{1}(x)=-e^{-x}$ is the exponential utility,

$$
J(u)=E\left[\int_{0}^{T}-e^{-\int_{0}^{t} \beta_{s} d s} e^{-u_{t} Y_{t}} \lambda_{t} d t+K Y_{0}\right] .
$$

Several features distinguish our optimal control problem from other stochastic control problems applied in finance and business, and these features make our problem more complicated to solve:

- Unlike the investment-consumption proposed by Merton [1974], the state variable $Y$ in our problem follows a BSDE rather than the usual SDE.

- Our objective functional $J(u)$ relies on the initial value $Y_{0}$ instead of the terminal value $Y_{T}$.

- The control variable $u$ enters nonlinearly into both the state equation and the objective functional.

$\left({ }^{4}\right)$ Here, the preference is a minor modification to the standard quasi-linear one in that the utility of $u_{t} Y_{t}$ is in its integration form over $[0, T]$. 
As mentioned before, to be more realistic, sometimes we can also investigate the following objective functional:

$$
J(L)=E\left[\int_{0}^{\tau} e^{-\int_{0}^{t} \beta_{s} d s} U_{1}\left(L_{t} Y_{t}\right) \lambda_{t} d t+U_{2}\left(Y_{0}\right)\right],
$$

where $\tau$ is the default time to characterize the firm's preference. Such an objective functional involves random time duration which makes its optimization problem more complicated. Here, we just sketch the key point towards its solution and postpone the full details to our future work: analogous to the proof of Theorem 3.1, we can transform the integration of the above functional into some integration with fixed time interval $[0, T]$; as a tradeoff, we need to introduce some extra terms with respect to $\lambda_{t}$ into our integrand.

4.2. Stochastic maximum principle. To ease the notation, hereafter we denote $-U_{1}$ by $l,-U_{2}$ by $\gamma$, and rewrite the optimal recovery problem as

$$
\inf _{u \in \mathcal{U}}-J(u)=\inf _{u \in \mathcal{U}} E\left[\int_{0}^{T} e^{-\int_{0}^{t} \beta_{s} d s} l\left(u_{t} Y_{t}\right) \lambda_{t} d t+\gamma\left(Y_{0}\right)\right] .
$$

As a preliminary step to solve our problem, we introduce the Hamiltonian function $H:[0, T] \times \mathbb{R} \times \mathcal{C} \times \mathbb{R}^{+} \rightarrow \mathbb{R}$ defined as

$$
H(t, q, u, y)=q_{t}\left(r_{t}+\lambda_{t} u_{t}\right) y_{t}+e^{-\int_{0}^{t} \beta_{s} d s} l\left(u_{t} y_{t}\right) \lambda_{t}
$$

According to the stochastic maximum principle, the optimal recovery policy $u^{*}$ maximizes the Hamiltonian function as well. A key object in the stochastic maximum principle is the adjoint variable $q$, which is dual to the state variable $Y$ and its dynamics is defined via the dual equation

$$
\left\{\begin{array}{l}
-d q_{t}=H_{y}\left(t, q_{t}, u_{t}, Y_{t}\right) d t \\
q_{0}=-\gamma_{y}\left(Y_{0}\right)
\end{array}\right.
$$

Proposition 4.3 (Stochastic Maximum Principle, Peng [1993]). Suppose the admissible control domain $\mathcal{C}$ of the optimal recovery problem is convex, $\left(u^{*}, Y^{*}\right)$ is the optimal control pair, and $q^{*}$ the corresponding adjoint variable. Then the following necessary condition holds true almost surely:

$$
H_{u}\left(t, q_{t}^{*}, u_{t}^{*}, Y_{t}^{*}\right) \cdot\left(v-u_{t}^{*}\right) \geq 0 \quad \forall v \in \mathcal{C} .
$$

When we consider the situation in which the admissible control domain $\mathcal{C}$ is an open set, the admissible control will be an interior control and the above stochastic maximum principle will take a simpler form so we have the following result. 
THEOREM 4.4. If the admissible control domain $\mathcal{C}$ is open, then the optimal control pair $\left(u^{*}, Y^{*}\right)$ for the optimal recovery problem satisfies

$$
l^{\prime}\left(u_{t}^{*} Y_{t}^{*}\right)=\gamma_{y}\left(Y_{0}^{*}\right) e^{\int_{0}^{t}\left(\beta_{s}-r_{s}\right) d s} .
$$

Proof. The Hamiltonian function $H$ for our problem is

$$
H\left(t, q_{t}^{*}, u_{t}^{*}, Y_{t}^{*}\right)=q_{t}^{*}\left(r_{t}+\lambda_{t} u_{t}^{*}\right) Y_{t}^{*}+e^{-\int_{0}^{t} \beta_{s} d s} l\left(u_{t}^{*} Y_{t}^{*}\right) \lambda_{t} .
$$

Due to the stochastic maximum principle, the optimal control $u^{*}$ should satisfy

$$
H_{u}\left(t, q_{t}^{*}, u_{t}^{*}, Y_{t}^{*}\right) \cdot\left(v-u_{t}^{*}\right) \geq 0 \quad \forall v \in \mathcal{C},
$$

where $q^{*}$ is given by the dual equation $\left({ }^{5}\right)$

$$
\left\{\begin{array}{l}
-d q_{t}^{*}=H_{y}\left(t, q_{t}^{*}, u_{t}^{*}, Y_{t}^{*}\right) d t \\
q_{0}^{*}=-\gamma_{y}\left(Y_{0}^{*}\right) .
\end{array}\right.
$$

Note that

$$
\left\{\begin{array}{l}
H_{u}(t, q, u, y)=q_{t} \lambda_{t} y_{t}+e^{-\int_{0}^{t} \beta_{s} d s} l^{\prime}\left(u_{t} y_{t}\right) \lambda_{t} y_{t}, \\
H_{y}(t, q, u, y)=q_{t}\left(r_{t}+\lambda_{t} u_{t}\right)+e^{-\int_{0}^{t} \beta_{s} d s} l^{\prime}\left(u_{t} y_{t}\right) \lambda_{t} u_{t} .
\end{array}\right.
$$

Therefore

$$
\begin{aligned}
& \left\{\begin{array}{l}
-d q_{t}^{*}=q_{t}^{*}\left(r_{t}+\lambda_{t} u_{t}^{*}\right) d t+e^{-\int_{0}^{t} \beta_{s} d s} l^{\prime}\left(u_{t}^{*} Y_{t}^{*}\right) \lambda_{t} u_{t}^{*} d t, \\
q_{t}^{*}=-\gamma_{y}\left(Y_{0}^{*}\right),
\end{array}\right. \\
& \left(q_{t}^{*} \lambda_{t} Y_{t}^{*}+e^{-\int_{0}^{t} \beta_{s} d s} l^{\prime}\left(u_{t}^{*} Y_{t}^{*}\right) \lambda_{t} Y_{t}^{*}\right)\left(v-u_{t}^{*}\right) \geq 0 \quad \forall v \in \mathcal{C} .
\end{aligned}
$$

Because the control domain $\mathcal{C}=(-\infty, 1)$ is an open set, any point in $\mathcal{C}$ will be an interior point, so necessarily

$$
q_{t}^{*} \lambda_{t} Y_{t}^{*}+e^{-\int_{0}^{t} \beta_{s} d s} l^{\prime}\left(u_{t}^{*} Y_{t}^{*}\right) \lambda_{t} Y_{t}^{*}=0 .
$$

In view of $\lambda_{t} Y_{t}^{*}>0$,

$$
q_{t}^{*}+e^{-\int_{0}^{t} \beta_{s} d s} l^{\prime}\left(u_{t}^{*} Y_{t}^{*}\right)=0 .
$$

Recalling the dual equation, we have

$$
\left\{\begin{array}{l}
-d q_{t}^{*}=q_{t}^{*} r_{t} d t \\
q_{0}^{*}=-\gamma_{y}\left(Y_{0}^{*}\right)
\end{array}\right.
$$

Its solution is

$$
q_{t}^{*}=-\gamma_{y}\left(Y_{0}^{*}\right) e^{-\int_{0}^{t} r_{s} d s} .
$$

As $U_{1}$ is strictly concave, so $h=\left(-l^{\prime}\right)^{-1}=\left(U_{1}^{\prime}\right)^{-1}$ exists, we have

$$
u_{t}^{*} Y_{t}^{*}=h\left(e^{\int_{0}^{t} \beta_{s} d s} q_{t}^{*}\right)=h\left(-\gamma_{y}\left(Y_{0}^{*}\right) e^{e_{0}^{t}\left(\beta_{s}-r_{s}\right) d s}\right) .
$$

$\left({ }^{5}\right)$ The initial condition of the dual equation in Peng [1993] should be $-\gamma\left(Y_{0}^{*}\right)$. 
It follows that

$$
\left\{\begin{array}{l}
d Y_{t}^{*}=\left(r_{t} Y_{t}^{*}+\lambda_{t} h\left(e^{\int_{0}^{t} \beta_{s} d s} q_{t}^{*}\right) d t+Z_{t}^{*} d W_{t},\right. \\
Y_{T}^{*}=\xi
\end{array}\right.
$$

From Pardoux and Peng [1990], the above BSDE has a unique adapted solution $\left(Y_{t}^{*}, Z_{t}^{*}\right)$ and the optimal recovery policy $u^{*}$ will be

$$
u_{t}^{*}=\frac{h\left(e^{\int_{0}^{t} \beta_{s} d s} q_{t}^{*}\right)}{Y_{t}^{*}}
$$

To find the optimal recovery policy $u^{*}$, we need first to solve the BSDE for the optimal pair $\left(u^{*}, Y^{*}\right)$; for the technical background on the numerical solution of BSDE, the reader may refer to Ma et al. [2002]. Roughly, from the structure of the BSDE, we can see the optimal recovery policy is changing in the opposite direction to the bond price: the higher the bond price, the lower the recovery rate, and vice versa. Moreover, to make our optimal recovery policy $u^{*}$ implementable or admissible, we need $u_{t}^{*}<1$, that is,

$$
u_{t}^{*}=\frac{h\left(e^{\int_{0}^{t} \beta_{s} d s} q_{t}^{*}\right)}{Y_{t}^{*}}<1
$$

or equivalently

$$
Y_{t}^{*}>h\left(e^{\int_{0}^{t} \beta_{s} d s} q_{t}^{*}\right)
$$

Define

$$
K_{t}^{*}=Y_{t}^{*}-h\left(e^{\int_{0}^{t} \beta_{s} d s} q_{t}^{*}\right)>0 .
$$

Using the Itô formula, $K_{t}^{*}$ should satisfy the following BSDE:

$$
\left\{\begin{array}{l}
d K_{t}^{*}=\left[r_{t} K_{t}^{*}+\left(r_{t}+\lambda_{t}\right) h\left(S_{t}^{*}\right)-\left(\beta_{t}-r_{t}\right) h^{\prime}\left(S_{t}^{*}\right) S_{t}^{*}\right] d t+Z_{t}^{*} d W_{t}, \\
K_{T}^{*}=\xi-h\left(e^{\int_{0}^{T} \beta_{s} d s} q_{T}^{*}\right),
\end{array}\right.
$$

where

$$
S_{t}^{*}=e^{\int_{0}^{t} \beta_{s} d s} q_{t}^{*}=-\gamma_{y}\left(Y_{0}^{*}\right) e^{\int_{0}^{t}\left(\beta_{s}-r_{s}\right) d s} .
$$

From the comparison theorem for BSDE (see Peng [1990] for details), we get the following result which leads to $u_{t}^{*}<1$ immediately.

Proposition 4.5. The optimal solution $u^{*}$ satisfies $u^{*}<1$ whenever

$$
\left(r_{t}+\lambda_{t}\right) h\left(S_{t}^{*}\right)-\left(\beta_{t}-r_{t}\right) h^{\prime}\left(S_{t}^{*}\right) S_{t}^{*}<0,
$$

and

$$
K_{T}^{*}=Y_{T}^{*}-h\left(-\gamma_{y}\left(Y_{0}^{*}\right) e^{\int_{0}^{T}\left(\beta_{t}-r_{t}\right) d t}\right)>0 .
$$

In particular, when $\beta_{t}=r_{t}$, because $r_{t}+\lambda_{t}>0$, conditions (4.15) and (4.16) take a simpler form

$$
h\left(-\gamma_{y}\left(Y_{0}^{*}\right)\right)<0
$$


5. Examples. In this section, we discuss some interesting and insightful examples and show how to explore the optimal recovery policy using the general results we derived in the previous section.

5.1. Negative exponential utility. First consider the case when $U_{1}$ is a negative exponential utility and $U_{2}$ remains linear $\left({ }^{6}\right)$,

$$
U_{1}(x)=-e^{-x}, \quad U_{2}(y)=K y, \quad K>0 .
$$

Then the objective functional takes the form

$$
J(u)=E\left[\int_{0}^{T}-e^{-\int_{0}^{t} \beta_{s} d s} e^{-u_{t} Y_{t}} \lambda_{t} d t+K Y_{0}\right],
$$

and the corresponding optimal recovery problem is

$$
\inf _{u \in \mathcal{U}}-J(u)=\inf _{u \in \mathcal{U}} E\left[\int_{0}^{T} e^{-\int_{0}^{t} \beta_{s} d s} e^{-u_{t} Y_{t}} \lambda_{t} d t-K Y_{0}\right],
$$

where

- $l=e^{-\int_{0}^{t} \beta_{s} d s} e^{-u y} \lambda, \quad \gamma=-K y$,

- $H=q[(r+\lambda u) y]+e^{-\int_{0}^{t} \beta_{s} d s} e^{-u y} \lambda$.

The admissible control domain $\mathcal{C}=(-\infty, 1)$ is an open set, so the stochastic maximum principle becomes

$$
e^{\int_{0}^{t} \beta_{s} d s} q_{t}^{*}=-\gamma_{y}\left(Y_{0}^{*}\right) e^{\int_{0}^{t}\left(\beta_{s}-r_{s}\right) d s}=e^{u_{t}^{*} Y_{t}^{*}} .
$$

Therefore

$$
u_{t}^{*} Y_{t}^{*}=-\ln \left(-\gamma_{y}\left(Y_{0}^{*}\right) e^{\int_{0}^{t}\left(\beta_{s}-r_{s}\right) d s}\right)=h\left(e^{\int_{0}^{t} \beta_{s} d s} q_{t}^{*}\right) .
$$

From the comparison theorem for BSDE, or the properties of linear BSDE, we know that a sufficient condition to have $Y_{t}^{*} \leq 1$ is

$$
\left(r_{t}+\lambda_{t}\right) h\left(S_{t}^{*}\right)-\left(\beta_{t}-r_{t}\right) h^{\prime}\left(S_{t}^{*}\right) S_{t}^{*}<0 .
$$

Its market implications are as follows:

- If $\beta_{t}=r_{t}$, then the optimal recovery policy $u^{*}$ exists whenever

$$
\ln \left(-\gamma_{y}\left(Y_{0}^{*}\right)\right)>0
$$

that is,

$$
K>1 .
$$

This tells us that if the linear utility coefficient is $K>1$, the optimal recovery policy can be designed in the financial market. Such market characteristics become apparent when we consider the asymptotic

$\left({ }^{6}\right)$ We can generalize this to the case

$$
U_{1}(x)=-\kappa e^{\alpha x}, \quad \alpha, \kappa<0, \quad U_{2}(y)=K y, \quad K>0 .
$$


behavior, that is, let $K \rightarrow \infty$. In this limit case, the objective functional $J(u)$ is simplified by only considering the initial capital $Y_{0}$, and eliminating the instantaneous gain process $u_{t} Y_{t}$. It is obvious that the optimal control in this situation should be $u^{*}=0$, that is, the firm should compensate all the loss to the bond holder once the default occurs. This means the bond is default-free from the perspective of the bond holder. Keep in mind that the higher the initial price $Y_{0}$, the lower the YTM. Therefore, our result can be understood that if the issuer cares more (when $K>1$ ) about the initial price, then the optimal recovery policy can be dynamically designed in this market model; however, if the firm cares less about the initial price $Y_{0}$ (when $K<1$ ), and more about the instantaneous gain process, their preference style will prohibit them from finding the optimal recovery policy in such a market.

- If $\beta_{t}=r_{t}$, notice that

$$
u_{t}^{*} Y_{t}^{*}=h\left(S_{t}^{*}\right)=h\left(-\gamma_{y}\left(Y_{0}^{*}\right)\right)=-\ln (K) .
$$

Of course, the price process satisfies $Y_{t}^{*}>0$, so the above equality tells us that

$$
u_{t}^{*}=\frac{-\ln (K)}{Y_{t}^{*}}<0
$$

Hence, another market implication is as follows: recall the financial meaning of $u_{t}^{*}=L_{t}^{*}$ is just the loss rate in RMV scheme, therefore, the firm should choose a negative recovery policy to maximize its utility. The negative recovery policy here means the firm should make more compensation than the loss to the bond holder once the default occurs. Further, the bigger the linear coefficient $K$, the greater the compensation. The total amount compensated to the bond holder when the default occurs at time $t$ will be $u_{t}^{*} Y_{t}^{*}=\ln (K)$, which is independent of the default time $\tau=t$.

- On the other hand, note that

$$
U_{1}(x)=-e^{-x}, \quad U_{2}(y)=K y, \quad K>0 .
$$

Correspondingly,

$$
U_{1}^{\prime}(x)=e^{-x}, \quad U_{2}^{\prime}(y)=K, \quad K>0 .
$$

As $x, y \rightarrow \infty, U_{1}^{\prime}(x) \rightarrow 0$ while $U_{2}^{\prime}(y) \equiv K>0$. Based on this observation, we know that, compared to the instantaneous gain process $u_{t} Y_{t}$ with utility $U_{1}$, the bond issuer cares more about the initially raised money $Y_{0}$ with $U_{2}$ as its utility. Consequently, the firm would like to adopt the negative recovery policy $u_{t}<0$ to minimize its discounted rate $r_{t}+\lambda_{t} u_{t}$, or equivalently, maximize $Y_{0}$. In fact, if the bond issuer commits himself to making more compensation than the loss to the 
bond holder whenever the default occurs, then the bond itself will be more "creditable" or "safer" than the risk-free treasure bond. In this case, the bond holder will only require $r_{t}+\lambda_{t} u_{t}<r_{t}$ as his return rate which is strictly less than the risk-free rate $r_{t}$. As a result, the firm will raise more money. In short, through adopting the negative recovery policy, the bond issuer succeeds in maximizing his utility where $U_{2}$ far outweighs $U_{1}$. The negative recovery policy is also discussed in Duffie and Singleton [1999].

5.2. Power utility. To complete the spectrum of important utility functions, in this subsection we will consider the power utility, which provides an interesting scenario different from that for the negative exponential utility:

$$
U_{1}(x)=\kappa x^{\alpha}, \quad 0<\alpha<1, \kappa>0, \quad U_{2}(y)=K y, \quad K>0 .
$$

To simplify the discussion, assume $U_{1}$ takes the special form

$$
U_{1}(x)=\frac{1}{p} x^{p}, \quad x \geq 0, p \in(0,1) .
$$

Remark 5.1. For the power utility, $p>0$ is called the Arrow-Pratt relative risk-aversion coefficient. If $p=1$, we define the log utility $U_{1}(x)=$ $\log x$ for $x>0$. If $x<0$, the utility is set to $-\infty$, thus effectively restricting $x$ from falling below zero.

The objective functional is

$$
J(u)=E\left[\int_{0}^{T} e^{-\int_{0}^{t} \beta_{s} d s} \frac{\left(u_{t} Y_{t}\right)^{p}}{p} \lambda_{t} d t+K Y_{0}\right],
$$

and the optimal recovery problem becomes

$$
\inf _{u \in \mathcal{U}}-J(u)=\inf _{u \in \mathcal{U}} E\left[\int_{0}^{T}-e^{-\int_{0}^{t} \beta_{s} d s} \frac{\left(u_{t} Y_{t}\right)^{p}}{p} \lambda_{t} d t-K Y_{0}\right] .
$$

Moreover, we know that

$$
\begin{aligned}
& \text { - } l=-e^{-\int_{0}^{t} \beta_{s} d s} \frac{(y u)^{p}}{p} \lambda, \quad p \in(-\infty, 1), p \neq 0, \gamma=-K y, \\
& \text { - } H=q[(r+\lambda u) y]-e^{-\int_{0}^{t} \beta_{s} d s} \frac{(y u)^{p}}{p} \lambda .
\end{aligned}
$$

So the stochastic maximum principle becomes

$$
\left(q_{t}^{*}-e^{-\int_{0}^{t} \beta_{s} d s}\left(u_{t}^{*} Y_{t}^{*}\right)^{p-1}\right)\left(v-u_{t}^{*}\right)>0 \quad \forall v \in \mathcal{U} .
$$

Because the admissible control domain $\mathcal{C}=(-\infty, 1)$ is open, we have

$$
e^{\int_{0}^{t} \beta_{s} d s} q_{t}^{*}=\left(u_{t}^{*} Y_{t}^{*}\right)^{p-1} .
$$


Then

$$
h\left(S_{t}^{*}\right)=u_{t}^{*} Y_{t}^{*}=\left[-\gamma_{y}\left(Y_{0}^{*}\right)\right]^{1 /(p-1)} \exp \frac{\int_{0}^{t}\left(\beta_{s}-r_{s}\right) d s}{p-1}>0 .
$$

To make the optimal recovery policy admissible, it is sufficient that

$$
\left(r_{t}+\lambda_{t}\right) h\left(S_{t}^{*}\right)-\left(\beta_{t}-r_{t}\right) h^{\prime}\left(S_{t}^{*}\right) S_{t}^{*}<0 .
$$

- If $\beta_{t}=r_{t}$, then

$$
\left(r_{t}+\lambda_{t}\right) h\left(S_{t}^{*}\right)<0
$$

This contradicts $h\left(S_{t}^{*}\right)>0$, hence the optimal recovery policy does not exist in this situation.

- Note that $h(x)=x^{1 /(p-1)}$, therefore (5.1) becomes

$$
\left[r_{t}+\lambda_{t}-\frac{\beta_{t}-r_{t}}{p-1}\right]\left(S_{t}^{*}\right)^{1 /(p-1)}<0 .
$$

As $S_{t}^{*}>0$, the optimal recovery exists whenever

$$
r_{t}+\lambda_{t}-\frac{\beta_{t}-r_{t}}{p-1}<0
$$

that is,

$$
r_{t}+\lambda_{t}<\frac{\beta_{t}-r_{t}}{p-1}
$$

If $r_{t}>0, \lambda_{t}>0, p-1<0$ are fixed, to make condition (5.2) hold true, it is necessary to have $\beta_{t}<r_{t}$. Once again, if $\beta_{t}=r_{t}$, it is impossible to design the optimal recovery policy due to the positivity of $r_{t}$ and $\lambda_{t}$.

6. Conclusion and extension. In this paper, we establish some important results concerning the credit risk pricing and the associated optimal recovery problem. Firstly, with the BSDE representation, we can fully explore the impact of the loss rate $L_{t}$ on the price of a credit-risky bond, as well as the objective functional involving the instantaneous gain process $u_{t} Y_{t}$ and initial value $Y_{0}$. Secondly, applying the stochastic maximum principle, we obtain the optimality condition for the optimal recovery problem, which possesses a significant econometric interpretation in financial market. Thirdly, we focus on two important cases where the utilities are negative exponential and power respectively. We determine the optimal recovery policy dynamically in these two cases and we propose some useful criteria to guarantee the implementation of the optimal recovery policy. It is remarkable that the procedures we employed here also work for general additive utility functions such as CARA, CRRA and HARA. In this respect, our work proposes a new framework that should be thought of as a basis for further practical applications. 
On the other hand, in this paper we concentrate on the optimal recovery problem where the state variable has BSDE evolution dynamics. The results we obtain can be generalized to the FBSDE case where the objective functional involves both the forward and backward variables. One illustrating example will be the case when $L$ takes small values; it will influence the firm's value $Y$, so some additional adjoint equation should be introduced. This will be the subject of future research. It would also be of interest to apply our technique to the problem of contract design in which the objective functional involves the stochastic differential utility (SDU) of Duffie and Epstein [1992].

\section{References}

P. Artzner and F. Delbaen (1995), Defaultable risk insurance and incomplete market, Math. Finance 5, 187-195.

T. Bielecki and M. Rutkowski (2002), Credit Risk: Modeling, Valuation and Hedging, Springer, Berlin.

J. M. Bismut (1973), Conjugate convex functions in optimal stochastic control, J. Math. Anal. Appl. 44, 383-404.

F. Black and M. Scholes (1973), The pricing of options and corporate liabilities, J. Political Economy 81, 637-654.

D. R. Cox (1955), Some statistical methods connected with series of events, J. Roy. Statist. Soc. Ser. B 17, 129-164.

D. Duffie and L. Epstein (1992), Stochastic differential utility, Econometrica 60, 353-394.

D. Duffie, M. Schroder and C. Skiadas (1996), Recursive valuation of defaultable securities and the timing of resolution of uncertainty, Ann. Appl. Probab. 6, 1075-1090.

D. Duffie and J. Singleton (1999), Modeling term structure of defaultable bonds, Rev. Financial Stud. 12, 687-720.

G. Hansen (1985), Indivisible labor and the business cycle, J. Monetary Economics 16, 309-328.

M. Harrison and D. Kreps (1979), Martingales and arbitrage in multiperiod security markets, J. Economic Theory 20, 381-408.

B. Hojgaard and M. Taksar (2001), Optimal risk control for a large corporation in the presense of returns on investment, Finance Stoch. 5, 527-547.

R. Jarrow and S. Turnbull (1995), Pricing derivatives on financial securities subject to credit risk, J. Finance 50, 53-84.

J. Ma, P. Protter, J. S. Martin and S. Torres (2002), Numerical methods for backward stochastic differential equations, Ann. Appl. Probab. 12, 302-316.

R. C. Merton (1974), On the pricing of corporate debt and the risk structure of interest rates, J. Finance 29, 449-470.

E. Pardoux and S. G. Peng (1990), Adapted solution of a backward stochastic differential equation, System Control Lett. 14, 55-61.

S. G. Peng (1990), A General Stochastic Maximum Principle for Optimal Control Problems, SIAM J. Control Optim. 28, 966-979.

S. G. Peng (1993), Backward stochastic differential equations and applications to optimal control, Appl. Math Optim. 27, 125-144.

P. Protter (1990), Stochastic Integration and Differential Equations, Springer, New York. 
R. Rogerson (1988), Indivisible labor, lotteries and equilibrium, J. Monetary Economics $21,3-16$.

N. Williams (2003), On dynamic principle-agent problems in continuous time, working paper.

CAB 632

Department of Mathematics and Statistics

University of Alberta

Edmonton, Alberta

Canada T6G 2G1

E-mail: jhuang@stat.ualberta.ca
Department of Real Estate and Construction The University of Hong Kong

Pokfulam, Hong Kong E-mail: h0499024@hkusua.hku.hk

Received on 2.5.2006;

revised version on 18.12.2006 
